\title{
Measuring the Dynamic Impedance of the Human Arm Without a Force Sensor
}

\author{
Matthew Dyck and Mahdi Tavakoli \\ Department of Electrical and Computer Engineering \\ University of Alberta \\ Edmonton, Alberta, Canada \\ Email: matthew.dyck@ualberta.ca, mahdi.tavakoli@ualberta.ca
}

\begin{abstract}
Rehabilitation robots may be used to accurately measure the mechanical impedance of the human arm in order to quantitatively assess the motor function of a patient undergoing neurorehabilitation therapy. However, the high cost of these robotic systems and their required sensors has posed a barrier to widespread clinical use. We present a technique to measure the mechanical impedance of the human arm without the need for a physical force sensor to measure human-robot interaction forces. Instead, these forces are accurately estimated by a virtual sensor that incorporates the robot's kinematics and dynamics, along with acceleration measurements from an inexpensive accelerometer. The identification techniques are validated on a mass-spring system of known impedance and are subsequently applied to data collected from the human arm.
\end{abstract}

Index Terms-force sensors, virtual sensors, accelerometers, arm impedance measurement, rehabilitation robotics

\section{INTRODUCTION}

Accurate measurement of the human arm's mechanical impedance is important in many areas of neurorehabilitation. Impedance, which describes the dynamic relationship between force and motion, may be quantified by measuring the reaction force the limb exerts as it is displaced by a robot. Three primary applications have motivated research in human arm impedance measurement to date: characterizing arm dynamics to design human-robot interaction systems [1], [2], investigating how the central nervous system (CNS) controls movement [3], [4], and assessing the motor function and muscle tone of impaired individuals undergoing rehabilitation therapy [5], [6]. The latter application is especially significant in a clinical context as it promises to augment conventional motor performance assessments based on therapist observation with accurate, objective data to monitor patient recovery and optimize therapy interventions.

Mussa-Ivaldi et al. [3] used a planar robot to impose step position perturbations on a human subject's hand, allowing the static endpoint impedance (i.e., stiffness) of the arm to be calculated in two Cartesian dimensions. Gomi et al. [4], Dolan et al. [2], and Tsuji et al. [7] extended this approach to measure not only the arm's stiffness, but also the dynamic impedance components of inertia and viscosity. Several researchers have since employed stochastic position or force perturbations to identify non-parametric models of arm impedance or admittance [5], [6]. Others have extended these techniques to analyze impedance in three dimensions [8], [9].
Detailed models of the arm's neuromusculoskeletal structure have been identified to distinguish impedance contributions of the arm's intrinsic biomechanics from those caused by its involuntary reflex responses [10]. Customized mechatronic systems for arm impedance measurement have also been designed to deliver high-bandwidth perturbations in multiple measurement planes [5] and accelerate data collection [11].

These methods for arm impedance measurement have all required a force sensor with the exception of the work by Mussa-Ivaldi et al. [3], which only measured the arm's static stiffness; they simply calculated the static human-robot interaction forces from the joint torques with knowledge of the robot's kinematics. Previous work in quantifying the arm's dynamic impedance, however, has relied on a force transducer to measure the changing interaction forces.

Given the cost-containment pressures faced by rehabilitation clinics, the relatively high retail cost of robotic therapy and diagnostic tools has presented a barrier to their widespread clinical use [12]. Commercial multiple degreeof-freedom (DOF) force/torque sensors typically retail for several thousand dollars due to their intricate strain-gauge design and precise calibration (e.g., a 6-DOF force/torque sensor from ATI Industrial Automation or JR3, Inc. costs approximately 6,000 USD). Unfortunately, these sensors have not become more affordable over the past decade and no low-cost alternative technology has appeared on the horizon. According to a recent survey of therapists, a complete upper limb rehabilitation robotic system should ideally retail for no more than 6,000 USD. While this target may be unrealistic for a comprehensive, multi-DOF rehabilitation robotic system, it underscores the importance of reducing the cost of this technology-especially when designing simpler, economical robotic devices intended for use in the patient's home. Thus, at least for the present, conventional multiple-DOF force/torque sensors are not suitable for inclusion in low-cost rehabilitation robotic systems. This paper contributes to previous work by developing and evaluating an economical human arm impedance measurement technique that does not require a physical force sensor. Instead, an accelerometer retailing for less than 15 USD is used to estimate interaction forces through a virtual sensor (or "soft sensor") derived from the robot's kinematics and dynamics.

This paper is organized as follows: Section II presents 
relevant mathematical formulae and introduces the experimental apparatus and data collection protocols, data analysis techniques are developed in Section III, results are discussed in Section IV, and concluding remarks follow in Section V.

\section{Methods And Materials}

In this paper, the arm's endpoint impedance is measured in two Cartesian dimensions as it interacts with the robot. This is sufficient to facilitate analysis of the relative contributions of the shoulder, elbow, and biarticular muscles to the overall limb impedance without necessitating the experimental complexity of a full three-dimensional measurement [13]. However, the techniques presented in this paper can be readily extended to three dimensions.

\section{A. Mathematical Preliminaries}

When a horizontal 2-DOF revolute-joint planar robot is subjected to an externally-applied force $F_{\text {ext }} \doteq\left[\begin{array}{ll}f_{x} & f_{y}\end{array}\right]^{T}$ at its end-effector, its movement is described by the following dynamics equation:

$$
\mathbf{I}(\Theta) \ddot{\Theta}+\mathbf{C}(\Theta, \dot{\Theta}) \dot{\Theta}+F_{r}(\dot{\Theta})=\tau_{r}+\mathbf{J}^{T} F_{\text {ext }}
$$

where $\mathbf{I}(\boldsymbol{\Theta})$ is the $2 \times 2$ inertia matrix, $\mathbf{C}(\boldsymbol{\Theta}, \dot{\boldsymbol{\Theta}})$ is the $2 \times 2$ Coriolis/centrifugal force matrix, $F_{r}$ is a $2 \times 1$ friction force vector, and $\mathbf{J}^{T}$ is the robot's Jacobian transpose matrix. Vectors $\Theta \doteq\left[\begin{array}{ll}\theta_{1} & \theta_{2}\end{array}\right]^{T}$ and $\tau_{r} \doteq\left[\begin{array}{ll}\tau_{1} & \tau_{2}\end{array}\right]^{T}$ represent joint angles and torques, respectively. Isolating $F_{\text {ext }}$ in this equation enables the external force to be calculated from records of the robot's motion in lieu of direct measurement by a force sensor: ${ }^{1}$

$$
F_{\text {ext }}=\left(\mathbf{J}^{T}\right)^{-1}\left(\tau_{r}-\mathbf{I}(\Theta) \ddot{\Theta}-\mathbf{C}(\Theta, \dot{\Theta}) \dot{\Theta}-F_{r}(\dot{\Theta})\right) .
$$

The human arm's endpoint impedance may be represented in a Cartesian plane by the following model:

$$
\mathbf{M} \ddot{X}+\mathbf{B} \dot{X}+\mathbf{K}\left(X-X_{v}\right)=-F_{\text {ext }},
$$

where $X=\left[\begin{array}{ll}x & y\end{array}\right]^{T}$ is the hand position, $X_{v}=\left[\begin{array}{ll}x_{v} & y_{v}\end{array}\right]^{T}$ is the hand's equilibrium position commanded by the CNS, and $F_{\text {ext }}$ is the force the hand exerts on the robot. The matrices

$$
\mathbf{M} \doteq\left[\begin{array}{ll}
m_{x x} & m_{x y} \\
m_{y x} & m_{y y}
\end{array}\right], \mathbf{B} \doteq\left[\begin{array}{ll}
b_{x x} & b_{x y} \\
b_{y x} & b_{y y}
\end{array}\right], \mathbf{K} \doteq\left[\begin{array}{ll}
k_{x x} & k_{x y} \\
k_{y x} & k_{y y}
\end{array}\right],
$$

which represent the hand inertia, damping, and stiffness, respectively, contain real-valued constants for the Cartesian plane of measurement. This model is derived from a simplified representation of the arm's neuromusculoskeletal structure as Dolan et al. detail in [2], but it may also be interpreted as a second-order Taylor-series linearization of an arbitrary nonlinear impedance model. Therefore, the model can accurately describe the lumped effects of the arm's intrinsic and reflexive

\footnotetext{
${ }^{1}$ While robotic control theory literature provides several methods for estimating external disturbance forces in real time (see [14] and [15] for examples), these causal, observer-based techniques suffer from convergence delays. For impedance measurement in the context of patient motor recovery assessment, non-causal offline analysis approaches may be used to achieve improved estimation accuracy.
}

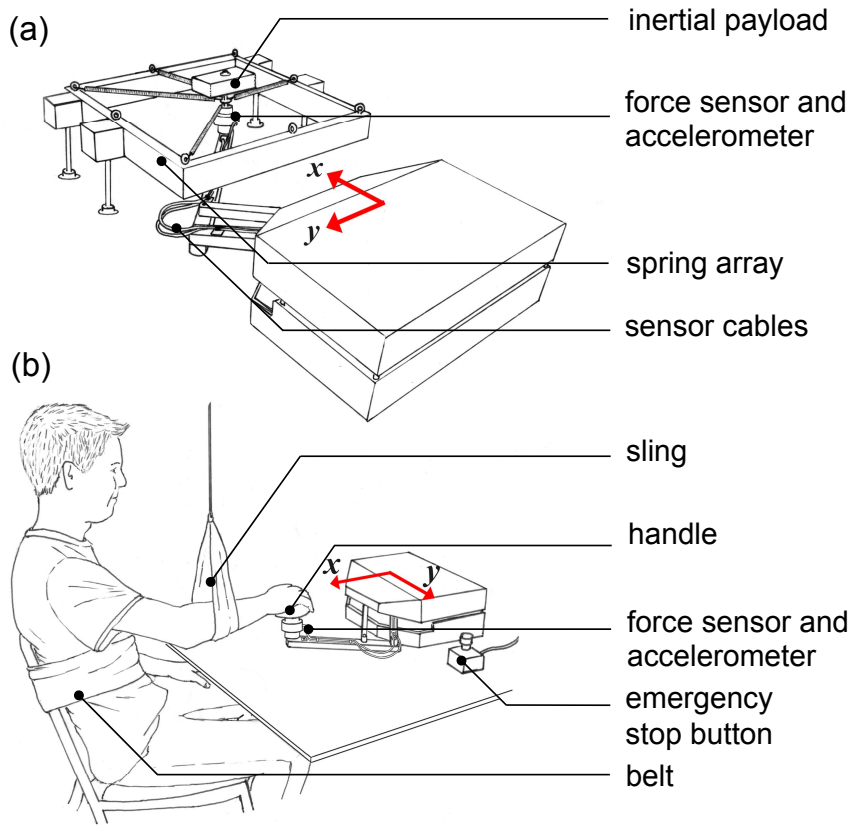

Fig. 1. Experimental apparatus for measuring impedance of (a) a spring array and an inertial payload that simulate the human arm, and (b) the actual human arm. Red arrows indicate the axes of the Cartesian coordinate system. In (b), measurements are performed in a horizontal plane approximately $10 \mathrm{~cm}$ below the shoulder joint.

impedance only for small perturbations about the constant equilibrium position $X_{v}$.

If the human arm is replaced by a mechanical system of known dynamic impedance comprising an inertial payload and a planar array of ideal springs connected to the robot's endeffector, (3) simplifies to

$$
\mathbf{M} \ddot{X}+\mathbf{K}\left(X-X_{0}\right)=-F_{\text {ext }},
$$

where $X_{0} \doteq\left[\begin{array}{ll}x_{0} & y_{0}\end{array}\right]^{T}$ is the equilibrium position of the spring configuration and $\mathbf{M}$ and $\mathbf{K}$ have the simplified structure

$$
\mathbf{M} \doteq\left[\begin{array}{cc}
m & 0 \\
0 & m
\end{array}\right], \quad \mathbf{K} \doteq\left[\begin{array}{cc}
k_{x x} & k_{s} \\
k_{s} & k_{y y}
\end{array}\right] \text {. }
$$

Note that the inertia matrix is diagonal with equal elements and the stiffness matrix is symmetric.

\section{B. Experimental Apparatus}

Human arm impedance measurements were performed with a 2-DOF planar rehabilitation robot (Fig. 1) manufactured by Quanser, Inc. (Markham, Ontario, Canada). This prototype device was expressly designed for home-based rehabilitation therapy, with an estimated retail cost of 15,000 USD [16]. The robot's capstan drive mechanism makes it readily backdrivable with low friction and inertia. At its end-effector, the robot can exert forces in excess of $50 \mathrm{~N}$ throughout its semicircular workspace, and the motors' optical encoders provide a Cartesian resolution of better than $0.002 \mathrm{~mm}$ in position measurement. Results of system identification experiments to identify the robot's dynamics are included in the 
Appendix. A two-axis accelerometer (ADXL-203, Analog Devices, Norwood, MA) was attached at the robot's end-effector to measure Cartesian accelerations. To compare the impedance measurements obtained through the robot's dynamics with those yielded by a conventional force measurement approach, a commercial 6-DOF force/torque sensor (50M31A3-125 DH, JR3 Inc., Woodland, CA) was also mounted on the robot's end-effector.

\section{Experimental Protocol}

The impedance measurement technique was first developed and validated by measuring the impedance of a mass-spring system intended to simulate a human arm with known inertia and stiffness. The technique was then applied to data collected from the actual human arm.

1) Impedance Measurement for a Mass-Spring System: The robot's end-effector was connected to an inertial payload and a planar array of linear mechanical springs (Fig. 1a). Although a two-dimensional spring array has a nonlinear stiffness field, the stiffness could be approximated as (5) for small perturbations about the equilibrium position of the spring system. By changing the number, stiffness, and arrangement of the springs included in the array, five different stiffness fields were obtained. For each of these spring arrangements, five different payloads were attached to the robot, creating a total of 25 unique impedance configurations. Table I lists the theoretical values of the $\mathbf{K}$ matrices (comprising $k_{x x}, k_{y y}, k_{s}$, and $X_{0}$ values calculated from independent measurements of the spring constants) and $\mathbf{M}$ matrices (comprising $m$ values measured by weighing the payloads).

To measure the impedance of each of the 25 configurations, the robot's end-effector was perturbed about the equilibrium position of the spring array. To create the perturbation, each of the robot's two joints was set to track a reference position comprising the sum of ten sinusoids with frequencies from 0 to $2 \mathrm{~Hz}$ using a proportional-derivative controller. The bandwidth of this perturbation signal was selected to match the experimental conditions under which the robot dynamic model was identified and validated and to avoid exciting the mechanical resonance of the mass-spring system at 4 to $5 \mathrm{~Hz}$. (Preliminary investigations revealed that the sustained resonant vibrations excited by higher frequency excitation signals caused the identified impedance to be overestimated.) Each joint moved through an angular range of $10^{\circ}$, causing the robot's end-effector to remain within a circular region $5 \mathrm{~cm}$ in diameter. All data logging and robot control actions occurred with a $1 \mathrm{kHz}$ sampling frequency. Three trials lasting $50 \mathrm{~s}$ each were performed for each of the 25 impedance configurations, yielding 75 trials in total.

2) Impedance Measurement for the Human Arm: For arm impedance identification, data was collected from a 23-yearold right-handed male with no history of motor impairment. The subject sat in front of the robot and rested his dominant hand on a hemispherical handle connected to the force sensor (Fig. 1b). (The interaction forces in this experiment were sufficiently small to allow the subject to simply rest his hand on the
TABLE I

THEORETICAL IMPEDANCE VALUES FOR MASS-SPRING SySTEM

\begin{tabular}{|c|c|c|c|c|c|c|c|}
\hline \multirow{2}{*}{$\begin{array}{l}\text { Stiffness } \\
\text { Matrix }\end{array}$} & \multicolumn{3}{|c|}{ Stiffness Values (N/m) } & \multicolumn{2}{|c|}{$\mathbf{X}_{0}(\mathbf{m m})$} & \multirow{2}{*}{$\begin{array}{l}\text { Inertia } \\
\text { Matrix }\end{array}$} & \multirow{2}{*}{$\frac{\text { Inertia value }(\mathbf{k g})}{m}$} \\
\hline & $k_{x x}$ & $k_{y y}$ & $k_{s}$ & $x_{0}$ & $y_{0}$ & & \\
\hline $\mathbf{K}_{1}$ & 421.57 & 213.35 & -18.87 & 364 & -9 & $\mathbf{M}_{1}$ & 0.539 \\
\hline $\mathbf{K}_{2}$ & 476.38 & 248.71 & -16.02 & 375 & -8 & $\mathrm{M}_{2}$ & 0.786 \\
\hline $\mathbf{K}_{3}$ & 239.54 & 421.29 & -12.52 & 394 & 53 & $\mathrm{M}_{3}$ & 1.031 \\
\hline $\mathbf{K}_{4}$ & 190.26 & 406.39 & -2.08 & 390 & 36 & $\mathrm{M}_{4}$ & 1.279 \\
\hline$K_{5}$ & 373.81 & 377.10 & 73.16 & 332 & -61 & $M_{5}$ & 1.527 \\
\hline
\end{tabular}

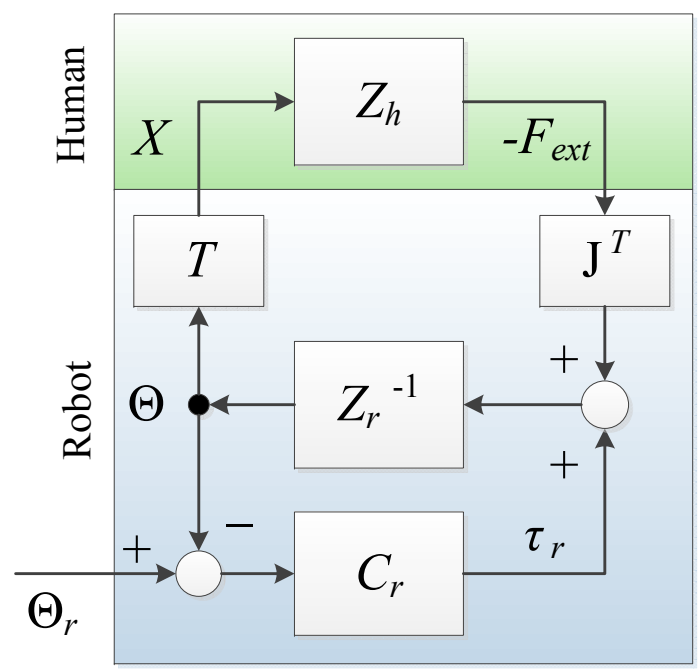

Fig. 2. Dynamics of the impedance measurement system. Robot and human (or mass-spring system) impedances are $Z_{r}$ and $Z_{h}$ respectively. The force sensor measures $F_{\text {ext }}$, the force exerted by the human on the robot. See text for definition of other variables.

robot's handle without the need to forcibly grasp the device.) The handle was positioned $50 \mathrm{~cm}$ anterior to the shoulder in the sagittal plane intersecting the shoulder joint. The subject's elbow was supported against gravity by a sling attached to a $2 \mathrm{~m}$ rope connected to the ceiling. A belt restrained the subject's torso to prevent translation of the upper body, which would have undermined the accuracy of the arm position measurements. The subject was instructed to relax his arm and avoid voluntarily exerting force while the robot perturbed his hand. This instruction was given to prevent voluntary force contributions from obscuring those arising from the subject's intrinsic impedance and reflex responses. In addition, the arm is usually similarly relaxed when traditional clinical assessments of muscle tone (e.g. the Modified Ashworth Scale [17]) are administered. Five trials were performed as the robot perturbed the hand in exactly the same manner as the massspring system.

\section{ANALYSIS}

During the data collection experiments, the positioncontrolled robot and the human arm formed a closed-loop system with the block diagram shown in Fig. 2. In this diagram, $Z_{r}$ and $Z_{h}$ represent the impedances of the robot and the human (or mass-spring system) respectively, and $C_{r}$ is 


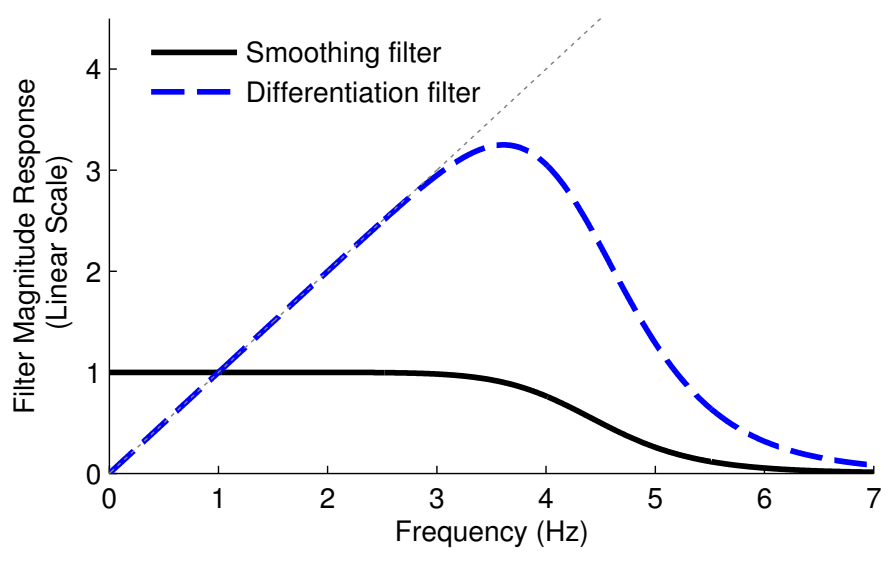

Fig. 3. Combined frequency response magnitude for the smoothing filter and differentiation filters each formed by a combination of Savitzky-Golay filtering and zero-phase Butterworth low-pass filtering. The ideal differentiator response is depicted by a thin dashed line.

the robot's joint-level position controller. The endpoint of the arm (or mass-spring system) was modelled in Cartesian space by (3) (or (5)) while the robot was modelled in joint space by (1). The two domains are related by the robot's forward kinematics $T$ and Jacobian-transpose $\mathbf{J}^{T}$.

The robot's position controller tracks a joint-domain perturbation signal $\Theta_{r}$, causing the robot to exert a torque $\tau_{r}$ that moves its joints to position $\Theta$ (close to $\Theta_{r}$ ). The joint position is translated into a Cartesian end-effector position through the robot's forward kinematics $T$, where the motion acts on the impedance $Z_{h}$ of the human arm's endpoint to generate a reaction force $-F_{\text {ext }}$ measured by the force sensor (note that $F_{\text {ext }}$ is defined as the force the human exerts on the robot). This force is translated into a joint-domain torque through the robot's Jacobian-transpose $\mathbf{J}^{T}$. This equivalent interaction torque together with the controller's torque output $\tau_{r}$ acts on the robot's admittance $Z_{r}^{-1}$ to complete the closedloop system. Since $F_{\text {ext }}=-Z_{h} X$, and both $F_{\text {ext }}$ and $X$ are measured, $Z_{h}$ can be identified directly from closed-loop data according to (3).

If $F_{\text {ext }}$ is not directly measured by a force sensor, the only other way it may be obtained is to use records of the robot's position $\Theta$ and command torque $\tau_{r}$ to work backwards through the robot's dynamics $Z_{r}$ and calculate $F_{\text {ext }}$ from the relation $-\mathbf{J}^{T} F_{\text {ext }}+\tau_{r}=Z_{r}^{-1} \Theta$, which is equivalent to (2). Therefore, elimination of the force sensor comes at the cost of requiring an accurate model of robot dynamics.

\section{A. Data Preprocessing}

Estimating arm impedance without direct measurement of $F_{\text {ext }}$ is not trivial, as the calculation of $F_{\text {ext }}$ from (2) requires accurate records of $\dot{\Theta}$ and $\ddot{\Theta}$. Finite-differencing and low-pass filtering of encoder data was found to be insufficiently noiserobust for this task. Instead, the accelerometer was introduced to allow direct measurement of acceleration. Furthermore, a Savitzky-Golay filter [18] was used to remove noise from all measured signals and calculate first time derivatives. This noncausal filtering technique fits a smooth $N^{t h}$-order polynomial
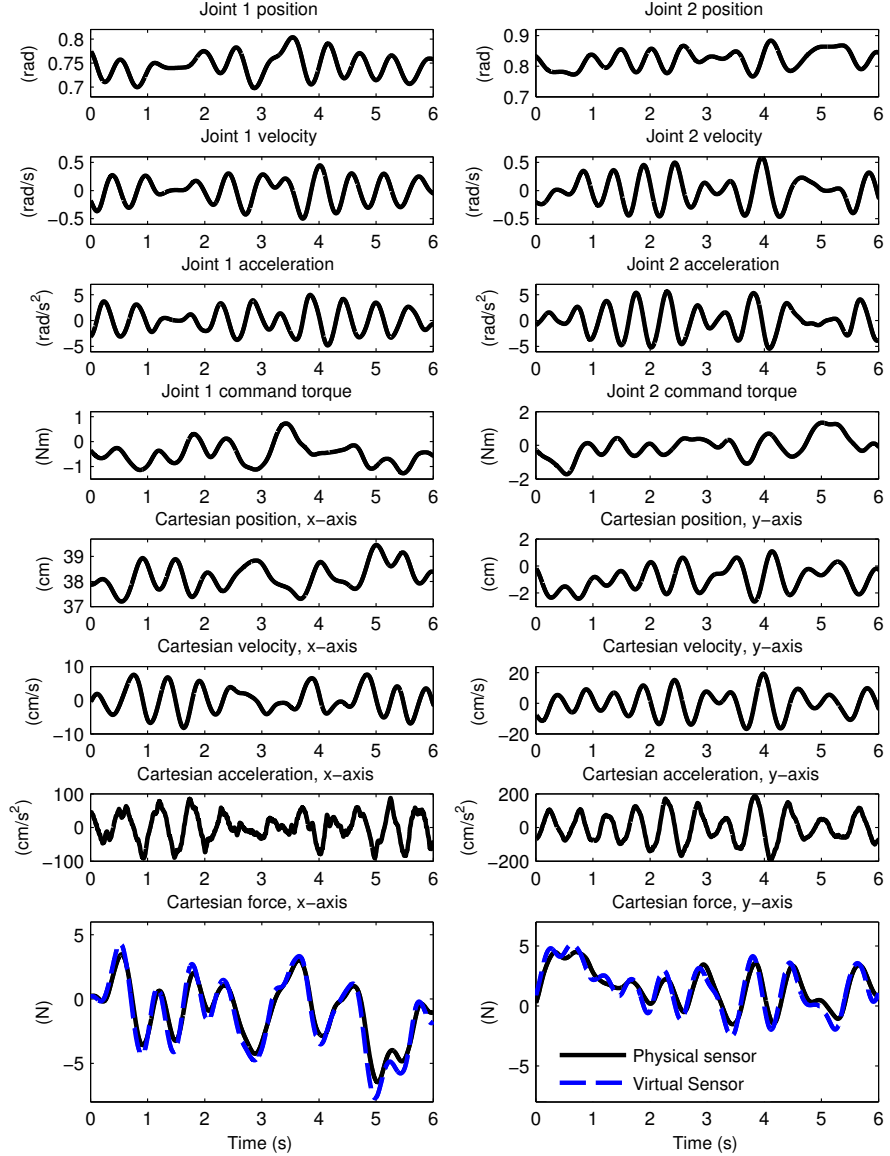

Fig. 4. A six-second segment of typical noise-filtered identification data for the mass-spring system with $\mathbf{K}_{\mathbf{5}}$ and $\mathbf{M}_{\mathbf{5}}$ as given in Table I. The source of each signal is listed in Table II. In the bottom row, the solid line shows the Cartesian force signal by the physical sensor and the dashed line indicates the corresponding force estimate of the virtual sensor.

to a window of $W$ consecutive data points. By ensuring $W \gg N$, the least-squares fitting process smooths out any random fluctuations in the unfiltered signal. The polynomial is evaluated and differentiated at the center of the window to yield both the smoothed signal and its first derivative at the corresponding time instant. The window is subsequently advanced forward by one sampling interval, and the algorithm is repeated until the entire signal has been processed by the filter. Through rigorous empirical evaluation, a filter with $N=6$ and $W=151$ was found to yield optimal results.

To enhance the noise-suppression characteristics of the Savitzky-Golay filter, its two outputs (i.e., the smoothed signal and its derivative) were passed through a zero-phase $5^{\text {th }}$-order Butterworth low-pass filter (MATLAB function filtfilt) with a $4.5 \mathrm{~Hz}$ cutoff frequency. Fig. 3 shows the magnitude response of combined Savitzky-Golay smoothing and Butterworth lowpass filtering operations (herein referred to as the "smoothing filter") and the combined Savitzky-Golay differentiation and Butterworth low-pass filtering operations (termed the "differentiation filter"). The smoothing filter maintains a magnitude response near unity over the $2 \mathrm{~Hz}$ bandwidth of the perturba- 
TABLE II

SignAL ORIGINS IN EACH IMPEDANCE IDENTIFICATION METHOD

\begin{tabular}{|c|c|c|c|c|c|c|c|c|}
\hline \multirow{2}{*}{ Method } & \multicolumn{8}{|c|}{ Impedance Identification Signals } \\
\hline & $X$ & $\dot{X}$ & $\ddot{X}$ & $\Theta$ & $\dot{\Theta}$ & $\ddot{\Theta}$ & $\tau_{r}$ & $F_{e x t}$ \\
\hline $\begin{array}{c}\text { Method 1 } \\
\text { (Proposed method) }\end{array}$ & $\begin{array}{l}\text { Forward } \\
\text { Kinematics }\end{array}$ & $\begin{array}{l}\text { Differentiation } \\
\text { Filter }\end{array}$ & Accelerometer & $\begin{array}{c}\text { Joint } \\
\text { Encoders }\end{array}$ & $\begin{array}{l}\text { Differentiation } \\
\text { Filter }\end{array}$ & Equation (7) & $\begin{array}{l}\text { Recorded from } \\
\text { Robot Controller }\end{array}$ & Equation (2) \\
\hline Method 2 & $\begin{array}{c}\text { Forward } \\
\text { Kinematics }\end{array}$ & $\begin{array}{l}\text { Differentiation } \\
\text { Filter }\end{array}$ & Accelerometer & $\begin{array}{c}\text { Joint } \\
\text { Encoders }\end{array}$ & (Not Used) & (Not Used) & (Not Used) & $\begin{array}{l}\text { Commercial } \\
\text { Force Sensor }\end{array}$ \\
\hline
\end{tabular}

tion signal, but drops to zero at higher frequencies for noise suppression. Similarly, the differentiation filter closely matches the ideal differentiator response from 0 to $2 \mathrm{~Hz}$, but decays to zero at higher frequencies. ${ }^{2}$

\section{B. Identification of Mass-Spring System Impedance}

The impedance of the mass-spring system was identified by fitting records of $F_{\text {ext }}, X$, and $\ddot{X}$ to (5) via linear least squares to obtain $\mathbf{M}, \mathbf{K}$, and $X_{0}$. All recorded signals (i.e., $\Theta$ from the joint encoders, $\ddot{X}$ from the accelerometer, $F_{\text {ext }}$ from the force/torque sensor, and the joint torque $\tau_{r}$ ) were passed through the smoothing filter to suppress noise prior to identification. The Cartesian position trajectory of the robot's end-effector was calculated from encoder data using the robot's forward kinematics, which were known precisely from manufacturer specifications. Table II summarizes the two methods used to obtain the human-robot interaction force:

Method 1 - Virtual sensor: The interaction force $F_{\text {ext }}$ was calculated from (2), with $\dot{\Theta}$ obtained from the differentiation filter and $\ddot{\Theta}$ calculated from the accelerometer's reading of $\ddot{X}$ according to

$$
\ddot{\Theta}=\mathbf{J}^{-1}(\ddot{X}-\dot{\mathbf{J}} \dot{\Theta}) .
$$

Method 2 - Physical sensor: $F_{\text {ext }}$ was directly measured by the force/torque sensor attached to robot's end-effector.

A segment of typical identification data for the mass-spring system is shown in Fig. 4. The first $40 \mathrm{~s}$ of each dataset were used for identification, while the final $10 \mathrm{~s}$ were used to validate the identification results through the Variance Accounted For (VAF) test statistic:

$$
V A F=100 \times\left(1-\operatorname{var}\left(f_{\text {ext }}-\hat{f}_{\text {ext }}\right) / \operatorname{var}\left(f_{\text {ext }}\right)\right),
$$

where $f_{\text {ext }}$ is the $x$ or $y$ component of $F_{\text {ext }}$ estimated by the virtual sensor or measured by the physical sensor and $\hat{f}_{\text {ext }}$ is the corresponding external force component predicted by the identified impedance model.

\section{Identification of Human Arm Impedance}

A handle with mass $\Lambda=135 \mathrm{~g}$ was present at the robot's end-effector during data collection for the human arm. To remove the effects of this handle from the identified inertia

\footnotetext{
${ }^{2}$ A Savitzky-Golay filter may also be used to calculate the second derivatives of $\Theta$ and $X$ directly, eliminating the need for an accelerometer. However, this approach was found to yield impedance estimates that did not agree with theoretical values. The inclusion of an inexpensive accelerometer notably enhanced the identification accuracy without compromising the goal of developing an economical impedance measurement technique.
}

matrix, the human arm's inertia, damping, and stiffness matrices were identified by applying the least-squares procedure to

$$
(\mathbf{M}+\Lambda \mathbf{I}) \ddot{X}+\mathbf{B} \dot{X}+\mathbf{K}\left(X-X_{v}\right)=-F_{\text {ext }},
$$

where $\mathbf{I}$ is the $2 \times 2$ identity matrix. The Cartesian velocity signal $\dot{X}$ was obtained from the differentiation filter. Interaction forces were calculated by applying the same two methods used for the mass-spring system. Again, the data sets were split into identification and validation portions and the VAF was calculated.

\section{RESUlts}

The bottom row of Fig. 4 demonstrates that the force estimated by the virtual sensor agreed favourably with that measured by the physical sensor. This enabled the proposed virtual sensor identification approach (Method 1) to yield impedance estimates comparable to those obtained with the physical sensor (Method 2) as discussed below.

\section{A. Results for the Mass-Spring System}

Table III shows the identified mass-spring system parameters obtained by Method 1 and Method 2. To simplify presentation, each entry represents the average and standard deviation of 15 trials. For instance, the stiffness values for $\mathbf{K}_{\mathbf{1}}$ are the average values obtained for the three trials performed for spring configuration $\mathbf{K}_{\mathbf{1}}$ with each of the five inertial payloads $\mathbf{M}_{1}$ through $\mathbf{M}_{5}$. Similarly, the inertia values reported for $\mathbf{M}_{1}$ are averages of the three trials performed for $\mathbf{M}_{\mathbf{1}}$ under each of the five spring arrangements. Identified equilibrium positions $X_{0}$ are omitted from the table for brevity as they were always within $18 \mathrm{~mm}$ of theoretical values.

Fig. 5 plots the identified parameters against their theoretical values listed in Table I. This figure shows that Method 1 and Method 2 yielded parameter estimates that were comparable to each other, demonstrating the feasibility of the virtual sensor approach (Method 1) for arm impedance measurement. Furthermore, both techniques produced parameter estimates close to theoretical values. While the agreement between the identified and theoretical inertia values was very strong, the agreement was slightly lower for stiffness values. This is partially due to approximations inherent in obtaining theoretical stiffness values through the numerical linearization of a nonlinear stiffness field calculated from empiricallydetermined spring constants.

Comparing the two identification methods against each other, the virtual sensor approach yielded stiffness parameters 
TABLE III

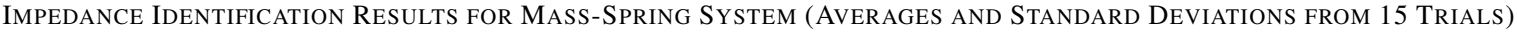

\begin{tabular}{|c|c|c|c|c|c|c|c|c|c|}
\hline \multirow{3}{*}{$\begin{array}{c}\text { Stiffness } \\
\text { Matrix }\end{array}$} & \multicolumn{6}{|c|}{ Identified Stiffness Values (N/m) } & \multirow{3}{*}{$\begin{array}{l}\text { Inertia } \\
\text { Matrix }\end{array}$} & \multicolumn{2}{|c|}{ Identified Inertia Values (kg) } \\
\hline & \multicolumn{3}{|c|}{ Method 1} & \multicolumn{3}{|c|}{ Method 2} & & \multirow{2}{*}{$\frac{\text { Method } 1}{m}$} & \multirow{2}{*}{$\frac{\text { Method } 2}{m}$} \\
\hline & $k_{x x}$ & $k_{y y}$ & $k_{s}$ & $k_{x x}$ & $k_{y y}$ & $k_{s}$ & & & \\
\hline$K_{1}$ & $504.43 \pm 3.62$ & $257.03 \pm 6.85$ & $-14.77 \pm 4.36$ & $458.99 \pm 3.26$ & $236.74 \pm 0.86$ & $-23.49 \pm 0.42$ & $M_{1}$ & $0.491 \pm 0.042$ & $0.544 \pm 0.015$ \\
\hline $\mathbf{K}_{2}$ & $569.98 \pm 13.74$ & $298.49 \pm 6.34$ & $-5.22 \pm 3.14$ & $514.03 \pm 3.6$ & $281.98 \pm 1.79$ & $-16.51 \pm 1.39$ & $\mathbf{M}_{2}$ & $0.719 \pm 0.042$ & $0.773 \pm 0.019$ \\
\hline $\mathbf{K}_{3}$ & $311.49 \pm 9.01$ & $436.13 \pm 2.58$ & $76.96 \pm 2.69$ & $275.06 \pm 1.22$ & $429.85 \pm 2.54$ & $-7.44 \pm 0.96$ & $\mathrm{M}_{3}$ & $0.95 \pm 0.034$ & $1.005 \pm 0.016$ \\
\hline $\mathbf{K}_{4}$ & $276.37 \pm 4.32$ & $434.26 \pm 4.25$ & $10.5 \pm 2.23$ & $226.94 \pm 0.91$ & $415.37 \pm 3.2$ & $-3.84 \pm 1.39$ & $\mathrm{M}_{4}$ & $1.156 \pm 0.041$ & $1.235 \pm 0.019$ \\
\hline $\mathbf{K}_{5}$ & $447.33 \pm 9.49$ & $382.45 \pm 4.38$ & $76.96 \pm 2.69$ & $417.24 \pm 1.92$ & $372.37 \pm 1.07$ & $70.11 \pm 1.35$ & $\mathrm{M}_{5}$ & $1.399 \pm 0.036$ & $1.471 \pm 0.019$ \\
\hline
\end{tabular}

TABLE IV

VAF BY METHOD $1\left(V A F_{x}, V A F_{y}\right)$

\begin{tabular}{cccccccc}
\hline $\begin{array}{c}\text { Stiffness } \\
\text { Matrix }\end{array}$ & $\begin{array}{c}\text { Max. Stiffness } \\
\text { Direction }\end{array}$ & $\mathbf{M}_{\mathbf{1}}$ & $\mathbf{M}_{\mathbf{2}}$ & $\mathbf{M}_{\mathbf{3}}$ & $\mathbf{M}_{\mathbf{4}}$ & $\mathbf{M}_{\mathbf{5}}$ \\
\hline \hline $\mathbf{K}_{\mathbf{1}}$ & $x$ & $97.5,93.5$ & $97.5,93.1$ & $97.5,92.6$ & $97.4,92.0$ & $97.2,92.1$ \\
$\mathbf{K}_{\mathbf{2}}$ & $x$ & $97.4,95.5$ & $97.7,95.2$ & $97.7,94.9$ & $97.5,93.6$ & $97.5,94.2$ \\
$\mathbf{K}_{\mathbf{3}}$ & $y$ & $88.7,96.7$ & $88.2,96.7$ & $87.6,96.6$ & $86.8,96.6$ & $84.9,96.7$ \\
$\mathbf{K}_{\mathbf{4}}$ & $y$ & $88.9,97.1$ & $88.2,97.1$ & $87.8,97.3$ & $83.5,96.2$ & $84.8,96.9$ \\
$\mathbf{K}_{\mathbf{5}}$ & N/A & $97.4,96.1$ & $97.3,95.7$ & $97.1,95.6$ & $97.1,95.4$ & $96.9,95.2$ \\
\hline
\end{tabular}
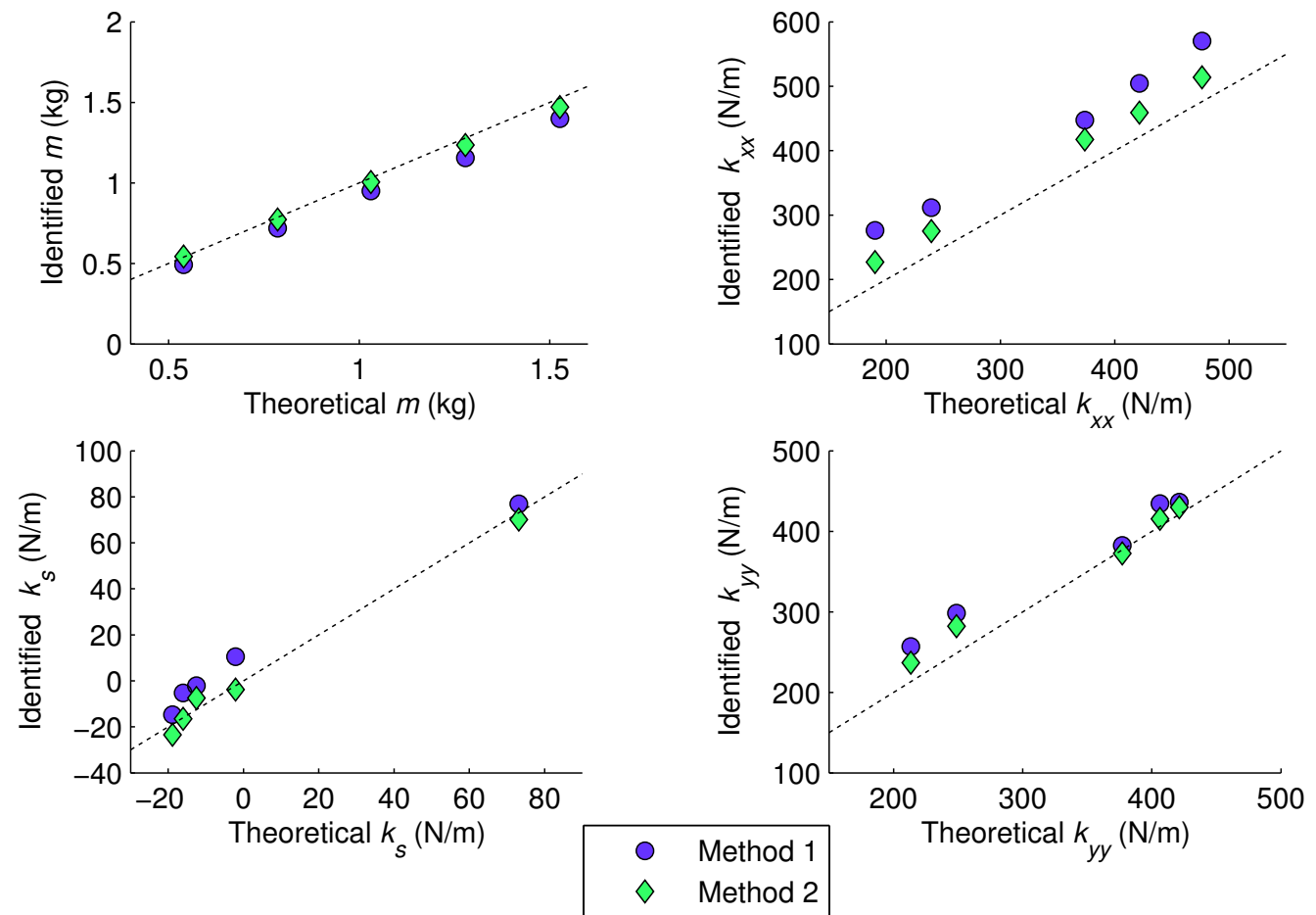

Fig. 5. Comparison of identified and theoretical impedance parameters for the mass-spring system. Data points in perfect agreement with theoretical values would lie on the dotted line.

that were slightly higher and inertia values that were slightly lower than those obtained with the physical sensor. These discrepancies are the result of the limited accuracy of the robot dynamics model obtained by system identification procedures. Equation (1) can be augmented to include a residual error force $F_{\text {err }}$ that accounts for any differences between the estimated robot dynamics obtained through system identification $(\hat{\mathbf{I}}(\Theta)$, $\hat{\mathbf{C}}(\Theta, \dot{\Theta})$, and $\left.\hat{F}_{r}(\dot{\Theta})\right)$ and their true values:

$$
\hat{\mathbf{I}}(\Theta) \ddot{\Theta}+\hat{\mathbf{C}}(\Theta, \dot{\Theta}) \dot{\Theta}+\hat{F}_{r}(\dot{\Theta})=\tau_{r}+\mathbf{J}^{T}\left(F_{\text {ext }}+F_{\text {err }}\right) \text {. }
$$

While a force sensor can accurately measure $F_{e x t}$ directly, calculating the external force through (2) actually yields the sum of $F_{\text {ext }}+F_{\text {err }}$. This error in the estimated force caused Method 1 to slightly underestimate inertia and overestimate stiffness relative to Method 2. Of all the identified parameters, the relative discrepancy between Methods 1 and 2 is the largest for $k_{x x}$. This indicates that $F_{\text {err }}$ contributed larger errors to $x$-component of the estimated force than the $y$-component-a reasonable finding since elastic forces in the force sensor and accelerometer cabling caused the robot to have the highest resistance to motion along the $x$ direction.

While the standard deviations yielded by Method 1 were larger than those obtained by Method 2, they were still very small in comparison to their corresponding mean values. This 
TABLE V

IMPEDANCE IDENTIFICATION RESULTS FOR HUMAN ARM

\begin{tabular}{|c|c|c|c|c|c|c|c|c|}
\hline \multirow{2}{*}{ Identification Method } & \multicolumn{2}{|c|}{ Inertia (kg) } & \multicolumn{2}{|c|}{ Viscosity (Ns/m) } & \multicolumn{2}{|c|}{ Stiffness $(N / m)$} & \multirow{2}{*}{$\begin{array}{c}\text { Equilibrium Position (mm) } \\
x_{v} \\
y_{v}\end{array}$} & \multirow{2}{*}{$\begin{array}{l}\text { VAF } \\
V A F_{x} \\
V A F_{y}\end{array}$} \\
\hline & $\begin{array}{l}m_{x x} \\
m_{y x}\end{array}$ & $\begin{array}{l}m_{x y} \\
m_{y y}\end{array}$ & $\begin{array}{l}b_{x x} \\
b_{y x}\end{array}$ & $\begin{array}{l}b_{x y} \\
b_{y y}\end{array}$ & $\begin{array}{l}k_{x x} \\
k_{y x}\end{array}$ & $\begin{array}{l}k_{x y} \\
k_{y y}\end{array}$ & & \\
\hline Method 1 & $\begin{array}{c}1.42 \pm 0.2 \\
0.69 \pm 0.11\end{array}$ & $\begin{array}{l}0.68 \pm 0.09 \\
0.99 \pm 0.07\end{array}$ & $\begin{array}{c}14.26 \pm 1.44 \\
1.08 \pm 0.79\end{array}$ & $\begin{array}{l}1.62 \pm 0.72 \\
6.82 \pm 0.75\end{array}$ & $\begin{array}{l}90.97 \pm 5.98 \\
25.92 \pm 4.58\end{array}$ & $\begin{array}{l}17.05 \pm 9.82 \\
27.22 \pm 4.46\end{array}$ & $\begin{array}{c}383 \pm 5 \\
15 \pm 9\end{array}$ & $\begin{array}{l}90.9 \\
90.2\end{array}$ \\
\hline Method 2 & $\begin{array}{l}1.45 \pm 0.21 \\
0.68 \pm 0.11\end{array}$ & $\begin{array}{l}0.65 \pm 0.09 \\
1.06 \pm 0.07\end{array}$ & $\begin{array}{c}10.88 \pm 1.12 \\
2.75 \pm 0.61\end{array}$ & $\begin{array}{l}3.09 \pm 0.53 \\
4.01 \pm 0.51\end{array}$ & $\begin{array}{l}72.34 \pm 6.99 \\
16.12 \pm 4.05\end{array}$ & $\begin{array}{l}6.29 \pm 10.14 \\
12.61 \pm 3.99\end{array}$ & $\begin{array}{c}382 \pm 5 \\
8 \pm 28\end{array}$ & $\begin{array}{l}91.3 \\
94.5\end{array}$ \\
\hline
\end{tabular}

indicates that the virtual sensor identification method showed excellent inter-trial reproducibility. The $k$ values identified for each stiffness matrix did not change as the inertial payload was increased, nor did the identified inertia values change with various spring configurations. Thus, both methods could effectively distinguish impedance contributions from the spring array and the inertial payload.

With Method 2, VAF values for both the $x$ and $y$ components of $F_{\text {ext }}$ were consistently over $99 \%$, demonstrating that the identified parameters described the impedance of the mass-spring system exceptionally well. Average VAF values obtained using Method 1 for each of the twenty-five impedance configurations are recorded in Table IV. Method 1 always yielded models with VAF values above $83 \%$, with the VAF being well over $92 \%$ in the vast majority of cases. This demonstrates that the proposed virtual sensor method also estimated the mass-spring system impedance with good accuracy.

The VAF values obtained by Method 1 also provide insight into the design of robotic devices that are well-suited to measuring impedance without a physical force sensor. As seen from Table I and indicated in Table IV, the stiffness matrices $\mathbf{K}_{\mathbf{1}}$ and $\mathbf{K}_{\mathbf{2}}$ had a much higher value in the $x$ direction than the $y$ direction. The opposite was true for $\mathbf{K}_{\mathbf{3}}$ and $\mathbf{K}_{\mathbf{4}}$, while $\mathbf{K}_{\mathbf{5}}$ had $k_{x x} \approx k_{y y}$. The VAF values obtained by Method 1 were always larger for the force component in the direction of greater stiffness. This occurred because the interaction force between the robot and the spring array system was larger in the direction of higher stiffness. When $F_{\text {ext }}$ is large, it overshadows any residual error forces contributed by inaccuracies in the robot dynamics $\left(F_{\text {ext }} \gg F_{\text {err }}\right)$. Therefore, a robot intended for impedance measurement with a virtual force sensor should be designed to have low impedance (e.g., it can be a back-drivable haptic device) such that the robot's motions are dominated by the externally-applied force rather than its intrinsic dynamics. In this case, any error in the identified dynamic matrices will give rise to force contributions that are small in comparison to the force exerted by the human arm, enabling a highly accurate identification of the arm's impedance.

\section{B. Results for the Human Arm}

Parameter values and standard deviations from the five human subject trials are presented in Table V. As with the mass-spring system, Methods 1 and 2 yielded very close inertia estimates. The viscosity and stiffness estimated by Method 1 were slightly larger but still close to those obtained by Method
2. VAF values are over $90 \%$ for both methods, indicating that the simple second-order model in (3) accurately captured the arm's dynamics for the relaxed grasping task in this study.

The inertia and viscosity values estimated for the human arm are comparable to those obtained in previous studies under similar experimental conditions by Dolan et al. [2] and Tsuji et al. [7], but the stiffness values are notably smaller. The high VAF values indicate that this discrepancy cannot be attributed to inaccurate identification. Rather, the lower apparent endpoint stiffness was likely due to the lowbandwidth, slowly varying perturbation signals used to identify arm dynamics. As discussed in the Appendix, flexibility in the robot's capstan drive mechanism enabled the robot dynamics model to be accurately identified only over the frequency range of 0 to $2 \mathrm{~Hz}$, and the perturbation signal used to identify the impedance of the human arm was necessarily limited to the frequencies over which the robot dynamics model was valid. Furthermore, the mass-spring system had a mechanical resonance between 4 and $5 \mathrm{~Hz}$, causing excessive vibrations that compromised identification accuracy when the system was excited in this frequency range. In contrast, Dolan et al. and Tsuji et al. both applied rapidly-rising step perturbations to the arm. The effective stiffness they measured may have been higher because these perturbations excited additional highfrequency impedance dynamics in the arm. Note that reflexive contributions to arm impedance have been found to become significant only at frequencies above $5 \mathrm{~Hz}$ [19]. In this work, forces from the arm's stiffness made smaller contributions to $F_{\text {ext }}$, explaining why the discrepancy between Methods 1 and 2 is higher for estimates of stiffness than for inertia or viscosity. If a suitable back-drivable robotic system were available with a dynamics model valid over a larger frequency range, the virtual sensor impedance measurement technique presented in this paper could be extended to identify higher frequency components of the human arm impedance.

\section{CONClusions ANd Future Work}

This paper demonstrates that arm impedance can be accurately measured without a force sensor if the humanrobot interaction forces are calculated using a virtual force sensor incorporating a valid model of the robot's dynamics. Although this technique necessarily entails a slightly higher estimation error compared to direct force measurement, it is a viable approach to add impedance measurement functionality to existing robotic devices without the need for potentially 
costly force/torque sensors. In a telerehabilitation scenario for instance, this approach could enable a rehabilitation therapist to remotely monitor a patient's arm impedance using an economical home-based rehabilitation robot.

This work focused on identifying a second-order Cartesian model of human arm impedance to demonstrate the feasibility of a virtual sensor approach for arm impedance measurement. The model was valid only for low frequencies due to bandwidth constraints imposed by the robot dynamics model and mechanical resonances in the mass-spring system used to validate the virtual sensor technique. Nevertheless, this virtual sensor technique may be extended to analyze the arm's impedance over a larger frequency range by applying it to a robot with a dynamic model valid at high frequencies, enabling separate analysis of the intrinsic and reflexive contributions to limb impedance. The virtual sensor approach could also could also be extended to other robotic therapy or assessment tasks that require offline analysis of force signals. For example, this approach could be used to measure impedance while the subject voluntarily co-contracts his muscles, to identify more complex models of neuromusculoskeletal system dynamics with the aid of higher-frequency perturbation signals, to quantify the mechanical work done during a therapy exercise, or to administer patient strength testing.

\section{APPENDIX}

\section{IDENTIFICATION OF ROBOT DYNAMICS}

The robot has the following dynamic matrices:

$$
\begin{gathered}
\mathbf{I}(\Theta)=\left[\begin{array}{cc}
\alpha_{1} & -1 / 2 \alpha_{2} \sin \left(\theta_{1}-\theta_{2}\right) \\
-1 / 2 \alpha_{2} \sin \left(\theta_{1}-\theta_{2}\right) & \alpha_{3}
\end{array}\right] \\
\mathbf{C}(\Theta, \dot{\Theta})=\left[\begin{array}{cc}
0 & 1 / 2 \alpha_{2} \cos \left(\theta_{1}-\theta_{2}\right) \dot{\theta_{2}} \\
1 / 2 * \alpha_{2} \cos \left(\theta_{1}-\theta_{1}\right) \dot{\theta_{1}} & 0
\end{array}\right] \\
F_{r}(\dot{\Theta})=\left[\begin{array}{c}
\alpha_{4} \dot{\theta_{1}} \\
\alpha_{5} \dot{\theta_{2}}
\end{array}\right]
\end{gathered}
$$

where $\alpha_{i}$ are constant parameters. To identify these parameters, each of the robot's two joints was set to track a signal comprising ten sinusoids to move each joint through a $40^{\circ}$ range such that the robot's end-effector covered the majority of its workspace. The bandwidth of the perturbation signal was limited to 0 to $2 \mathrm{~Hz}$, as higher-frequency excitations were found to compromise identification accuracy due to elasticity in the robot's capstan drive mechanism.

Six trials were performed, each lasting $50 \mathrm{~s}$ in duration. The first $40 \mathrm{~s}$ of data from each trial were used to identify parameters by linear least-squares. The identified model was validated against the final $10 \mathrm{~s}$ of data by applying the variance accounted for (VAF) test statistic. The following identified parameters consistently yielded VAF values in excess of $99 \%$ for $\tau_{1}$ and $90 \%$ for $\tau_{2}$.

\section{ACKNOWLEDGEMENT}

We gratefully acknowledge the support of the Natural Sciences and Engineering Research Council (NSERC) of Canada, Quanser Inc., and Alberta Innovates - Technology Futures.
TABLE VI

IDENTIFIED DyNAMIC PARAMETERS WITH STANDARD DEVIATIONS

\begin{tabular}{cc}
\hline Parameter & Value \\
\hline \hline$\alpha_{1}$ & $0.06929 \pm 0.00003$ \\
$\alpha_{2}$ & $0.04217 \pm 0.00007$ \\
$\alpha_{3}$ & $0.04416 \pm 0.00004$ \\
$\alpha_{4}$ & $0.06510 \pm 0.00176$ \\
$\alpha_{5}$ & $0.07389 \pm 0.00072$ \\
\hline
\end{tabular}

\section{REFERENCES}

[1] N. Hogan, "Controlling impedance at the man/machine interface," in Proc. 1989 Int. Conf. Robotics and Automation, 1989, pp. 1626-1631.

[2] J. Dolan, M. Friedman, and M. Nagurka, "Dynamic and loaded impedance components in the maintenance of human arm posture," IEEE Trans. Syst. Man Cybern., vol. 23, no. 3, pp. 698-709, 1993.

[3] F. A. Mussa-Ivaldi, N. Hogan, and E. Bizzi, "Neural, mechanical, and geometric factors subserving arm posture in humans," J. Neurosci. vol. 5, no. 10, pp. 2732-2743, Oct. 1985.

[4] H. Gomi, Y. Koike, and M. Kawato, "Human hand stiffness during discrete point-to-point multi-joint movement," in Proc. Annu. Int. Conf. IEEE Eng.in Medicine and Biology Soc., Oct. 1992, pp. 1628-1629.

[5] A. M. Acosta, R. F. Kirsch, and E. J. Perreault, "A robotic manipulator for the characterization of two-dimensional dynamic stiffness using stochastic displacement perturbations," J. Neurosci. Meth., vol. 102, no. 2, pp. 177-186, Oct. 2000.

[6] J. J. Palazzolo, M. Ferraro, H. I. Krebs, D. Lynch, B. T. Volpe, and N. Hogan, "Stochastic estimation of arm mechanical impedance during robotic stroke rehabilitation," IEEE Trans. Neural Syst. Rehabil. Eng., vol. 15, no. 1, pp. 94-103, Mar. 2007.

[7] T. Tsuji, P. G. Morasso, K. Goto, and K. Ito, "Human hand impedance characteristics during maintained posture," Biol. Cybern., vol. 72, no. 6, pp. 475-485, Jan. 1995.

[8] P. K. Artemiadis, P. T. Katsiaris, M. V. Liarokapis, and K. J. Kyriakopoulos, "Human arm impedance: Characterization and modeling in 3D space," in 2010 IEEE/RSJ Int. Conf. Intelligent Robots and Syst., Oct. 2010, pp. 3103-3108.

[9] K. Park and P. H. Chang, "Stochastic estimation of human shoulder impedance with robots: An experimental design," in IEEE Int. Conf. Rehabilitation Robotics, vol. 2011, Jan. 2011.

[10] E. de Vlugt, A. C. Schouten, and F. C. T. van der Helm, "Quantification of intrinsic and reflexive properties during multijoint arm posture," $J$. Neurosci. Meth., vol. 155, no. 2, pp. 328-349, Sep. 2006.

[11] L. Masia, G. Sandini, and P. G. Morasso, "A novel mechatronic system for measuring end-point stiffness: Mechanical design and preliminary tests," in IEEE Int. Conf. Rehabilitation Robotics, vol. 2011, Jan. 2011.

[12] M. Lee, M. Rittenhouse, and H. A. Abdullah, "Design issues for therapeutic robot systems: Results from a survey of physiotherapists," J. Intell. Robot. Syst., vol. 42, no. 3, pp. 239-252, Mar. 2005.

[13] N. Hogan, "The mechanics of multi-joint posture and movement control," Biol. Cybern., vol. 52, no. 5, pp. 315-331, Sep. 1985.

[14] A. Radke, "A survey of state and disturbance observers for practitioners," in 2006 Amer. Control Conf., no. 2, 2006, pp. 5183-5188.

[15] A. Mohammadi, M. Tavakoli, H. Marquez, and F. Hashemzadeh, "Nonlinear disturbance observer design for robotic manipulators," Control Eng. Pract., vol. 21, no. 3, pp. 253 - 267, 2013.

[16] E. C. Lu, "Development of an upper limb robotic device for stroke rehabilitation," Master's thesis, University of Toronto, 2011.

[17] C. Bosecker, L. Dipietro, B. Volpe, and H. I. Krebs, "Kinematic robotbased evaluation scales and clinical counterparts to measure upper limb motor performance in patients with chronic stroke," Neurorehab. Neural Re., vol. 24, no. 1, p. 6269, Jan 2010.

[18] A. Savitzky and M. J. E. Golay, "Smoothing and differentiation of data by simplified least squares procedures," Anal. Chem., vol. 36, no. 8, pp. 1627-1639, Jul. 1964.

[19] A. C. Schouten, E. D. Vlugt, and F. C. T. van der Helm, "Design of perturbation signals for the estimation of proprioceptive reflexes." IEEE Trans. Biomed. Eng., vol. 55, no. 5, pp. 1612-1619, May 2008. 